\title{
Modified Chest Wall Pulley for Lung Excision during Single-Incision Thoracoscopic Surgery for Primary Spontaneous Pneumothorax
}

\author{
Kenji Tsuboshima, MD, ${ }^{1}$ Machiko Nagata, MD, ${ }^{1}$ Teppei Wakahara, MD, ${ }^{1}$ \\ Yasumi Matoba, MD, $\mathrm{PhD},{ }^{1}$ and Yoshimasa Maniwa, $\mathrm{MD}, \mathrm{PhD}^{2}$
}

\begin{abstract}
We reported the feasibility of single-incision thoracoscopic surgery bullectomy using a chest wall pulley for lung excision (PulLE) in patients with primary spontaneous pneumothorax (PSP). PulLE has many merits including comfort of manipulation, cosmetic advantages, etc., compared to other procedures. However, our method was utilized for relatively straightforward cases. The PulLE was contraindicated for PSPs with multiple or comprehensive bullae. Therefore, we developed the modified PulLE (mPulLE) to treat such cases in February 2015. Although one chest wall pulley is placed in the thoracic cavity for a PulLE, two are used for an mPulLE. Herein, we describe the mPulLE technique.
\end{abstract}

Keywords: PulLE, SITS, pneumothorax, modified

\section{Introduction}

Recently, the feasibility of single-incision thoracoscopic surgery (SITS) was reported because of cosmetic benefits and lower invasiveness. ${ }^{1-4)}$ We reported the use of SITS with a chest wall pulley for lung excision (PulLE) in patients with primary spontaneous pneumothorax (PSP), and compared this technique with conventional three-port video-assisted thoracic surgery (VATS). ${ }^{5)}$ PulLE, which needs no special device, has many merits, including comfort of manipulation, cosmetic advantages, and lower cost, compared to other procedures. However, our indication for this method was relatively narrow, and complex cases with multiple or comprehensive bullae

${ }^{1}$ Department of Thoracic Surgery, Takasago Municipal Hospital, Takasago, Hyogo, Japan

${ }^{2}$ Division of Thoracic Surgery, Kobe University Graduate School of Medicine, Kobe, Hyogo, Japan

Received: May 23, 2016; Accepted: July 30, 2016

Corresponding author: Kenji Tsuboshima, MD. Department of Thoracic Surgery, Takasago Municipal Hospital, 33-1 Kamimachi, Arai Town, Takasago, Hyogo 676-8585, Japan

Email: drniwatori@smn.enjoy.ne.jp

(C)2016 The Editorial Committee of Annals of Thoracic and Cardiovascular Surgery. All rights reserved. were excluded. Operating on such PSPs is relatively easy using conventional VATS. Therefore, we developed the modified PulLE (mPulLE) to treat these PSP cases in February 2015. Only one chest wall pulley is placed in the thoracic cavity for PulLE, while two are placed for mPulLE. Herein, we describe the mPulLE technique. For Fig. 1, Copyright (C) 2016 Johnson \& Johnson K.K. all rights reserved. This study was approved by the Institutional Review Board of the Takasago Municipal Hospital.

\section{Technique}

The PulLE procedure

1) Thoracic cavity observation

As reported previously, $\left.{ }^{5}\right)$ the patient was placed in a lateral position under general anesthesia. An 18-mm skin incision was performed at the sixth intercostal space (ICS) at the median axillary line and a LAPPROTECTOR mini-mini (Hakko, Japan) was placed. The thoracic cavity was observed using a 5-mm $30^{\circ}$ thoracoscope. When a single lesion was easily identified, we decided to perform PulLE.

2) Traction suturing of the lung

Visceral pleura near the bulla was sutured for traction using 2-0 nylon thread. 
(a)

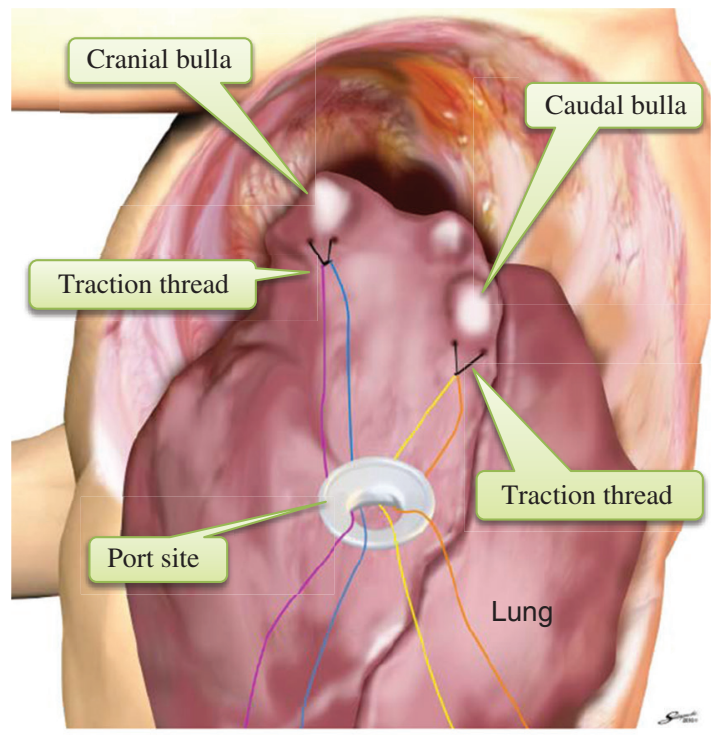

(c)

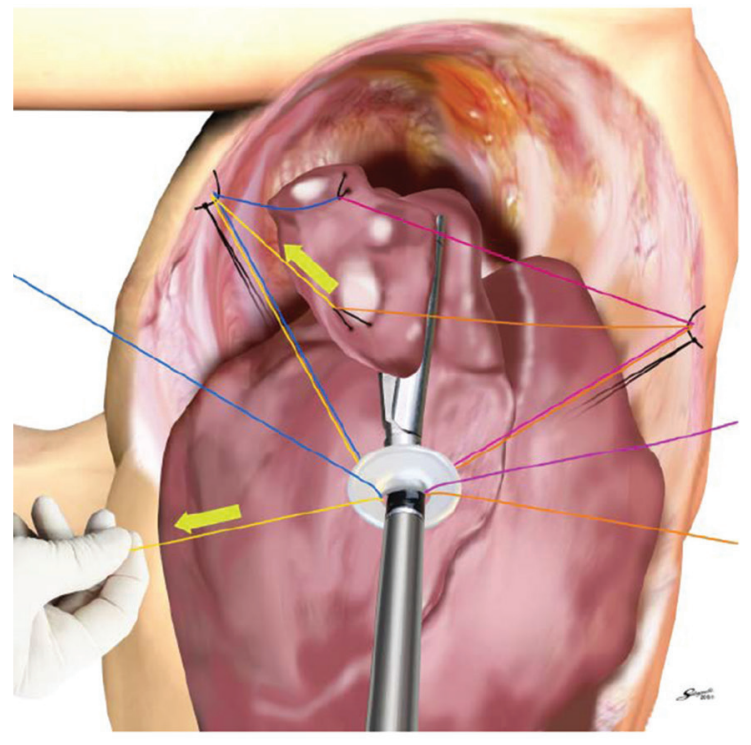

(b)

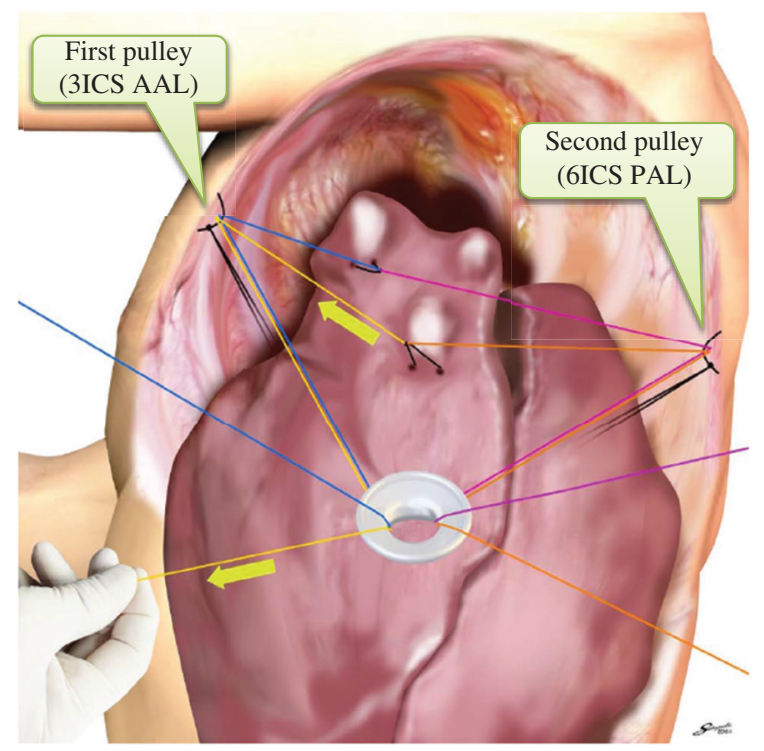

(d)

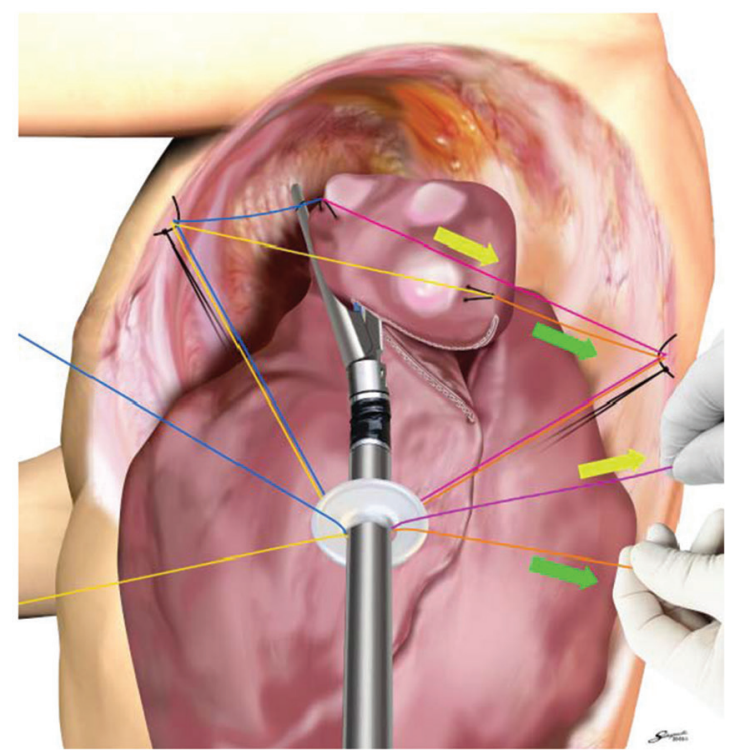

Fig. 1 Schema of single-incision thoracoscopic surgery using a chest wall pulley for lung excision in primary spontaneous pneumothorax treatment. (a) Suturing near the caudal and cranial bullae traction threads. (b) Constructing the first and second pulley involves two traction threads and a supporting thread at the third intercostal space (ICS) at the anterior axillary line (AAL) and the sixth ICS at the posterior axillary line (PAL). $(\mathbf{c}, \mathbf{d})$ Manipulation of four traction threads for resecting the lesion in the direction of the arrow.

3) Chest wall pulley

Parietal pleura from the thoracic cavity were sutured at the third ICS level at the anterior axillary line by another 2-0 nylon thread to serve as a chest wall pulley. This thread is known as a supporting thread. The supporting thread was wound around the traction thread, out of the thoracic cavity, and ligated to the thoracic cavity with a pusher. The pulley was formed from both traction and supporting threads.

4) Bulla resection

Manipulating the traction thread outside the thoracic cavity allowed the lesion to be moved partially toward the best position for excision using stapler (Powered ECHELON FLEX, JJKK, USA). The lung's 

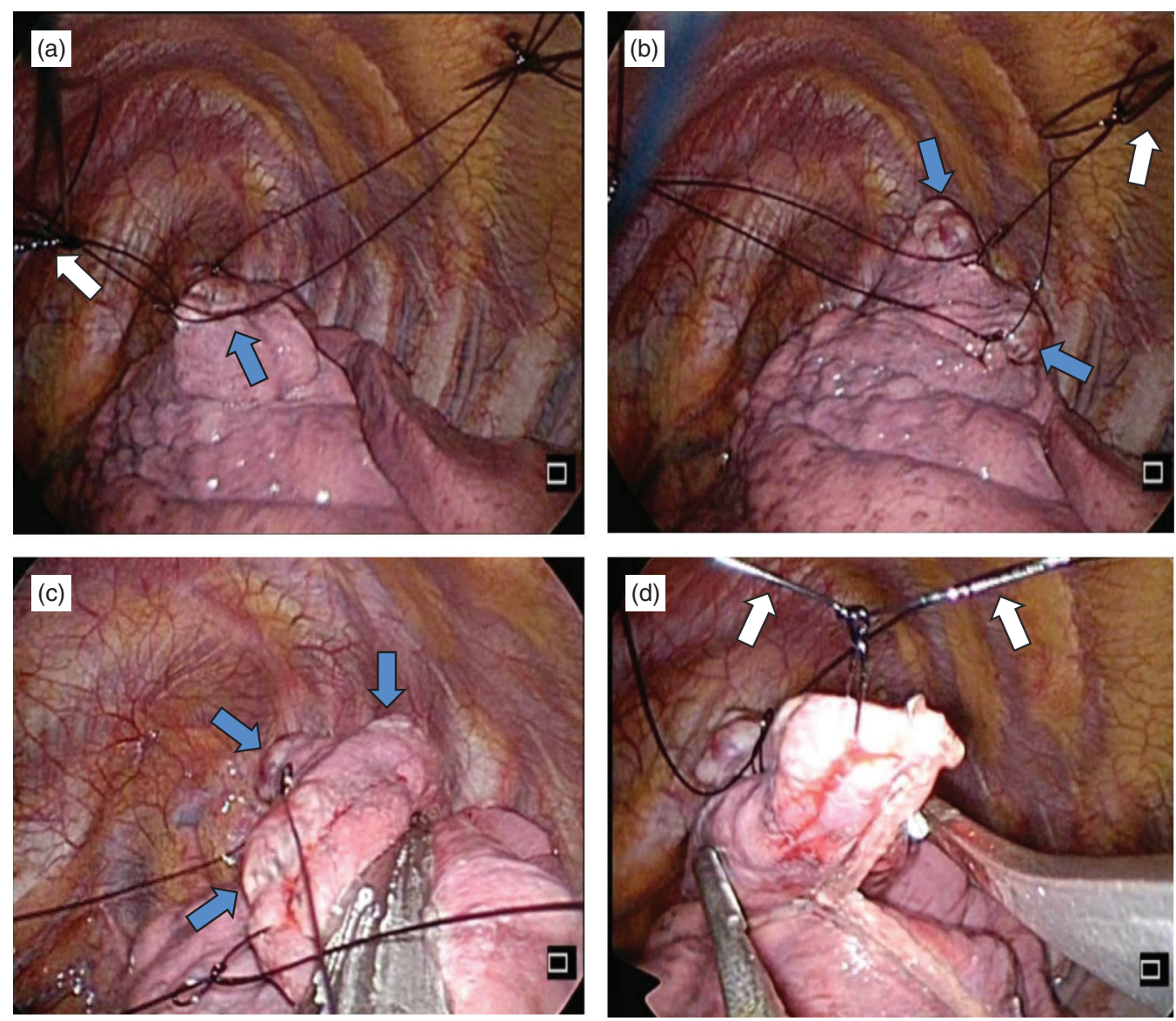

Fig. 2 Intraoperative findings of single-incision thoracoscopic surgery using a modified chest wall pulley for lung excision. (a) Traction of the caudal bulla (blue arrows) to the first pulley (white arrow). (b) Traction of the cranial bulla (blue arrows) to the second pulley (white arrow). (c) Resection of the bulla (blue arrow) using stapler. (d) Fine staple line findings using traction threads (white arrows) for both the first and second pulleys.

staple line was reinforced with an oxidized cellulose sheet.

\section{The mPulLE procedure}

If multiple or comprehensive bullae were found in the thoracic cavity observation, we performed mPulLE as follows:

1) Traction suturing of the lung

Visceral pleura near the caudal and cranial bullae was sutured for traction sequentially from the bottom using 2-0 nylon threads (Fig. 1a).

2) First chest wall pulley

Parietal pleura at the third ICS level at the anterior axillary line was sutured from the thoracic cavity to serve as the first chest wall pulley by another 2-0 nylon thread, and the caudal and cranial traction threads were then passed through the supporting thread one by one as with PulLE (Fig. 1b).

3) Second chest wall pulley

Similarly, the second pulley was placed at the sixth ICS of the posterior axillary line. The residual threads were passed through the supporting thread one by one (Fig. 1b).

4) Bulla resection

Manipulating four traction threads out of the thoracic cavity simultaneously allowed the lesion to be moved to an arbitrary site (Figs. 1c, 1d and 2). Then, as with the PulLE, the lesion was resected.

\section{Patient characteristics and surgical results}

Between February 2012 and March 2016, PulLE and mPulLE were performed in 57 and 13 cases, respectively, 
out of 172 consecutive PSP cases undergoing VATS in our hospital. We retrospectively compared both procedures in terms of patient backgrounds and results of short-term operations.

The number of bullae was $2.1 \pm 1.2$ and $3.0 \pm 1.6$, respectively $(\mathrm{p}=0.02)$. On the other hand, operative times were $66.7 \pm 19.0 \mathrm{~min}$ and $74.9 \pm 16.5 \mathrm{~min}$, and the number of staplers was $3.4 \pm 0.9$ and $3.8 \pm 1.2$, respectively. There were no significant differences between the groups for factors like age, gender, smoking habit, body mass index, and the aforementioned surgical results. Neither group had serious complications. Although the follow-up period was relatively short, there was no postoperative recurrence for mPulLE. Statistical analyses were conducted using the Student-t, Welch, and $\chi^{2}$ tests. A P-value of less than 0.05 was considered to be statistically significant. All tests were performed using JMP statistical software (version 9, SAS, USA).

\section{Discussion}

The feasibility of SITS has been reported recently. ${ }^{1-7)}$ Yamamoto and colleagues first described SITS bullectomy using an original scope guide. ${ }^{1)}$ We previously reported the feasibility of SITS using a PulLE. ${ }^{5)}$ Movement of the lesion using the traction thread for a PulLE allows lower interference from other devices than in other SITS techniques. However, the movement is directed toward the chest wall pulley and the moving distance is relatively short. Therefore, it was difficult to resect multiple and comprehensive bullae with a PulLE. It is possible to lead the bulla in any direction using four traction threads simultaneously using the mPulLE. Moreover, the lesion can be moved to a longer distance than with a PulLE because the second chest wall pulley is more remote from the lesion than the first pulley. Hence, it is easy to observe and resect multiple or comprehensive bullae using the mPulLE.

Because additional suturing at the visceral and parietal pleurae required only a few minutes, there was no significant difference in operative time. For multiple or wide bullae, the resected volume increases, requiring many staplers. However, there was no significant difference in the number of staplers. This may be because of the reduction in extra resection to tract the lesion to an optimal position for excision. We will continue to perform this technique and investigate the long-term results in the future.

Our mPulLE procedure facilitates SITS for PSP cases with multiple or comprehensive bullae. Because our procedure is simple, requiring no special devices which are expensive, and the cosmetic results are favorable, we believe our method to be a new standard treatment for PSP with multiple or comprehensive bullae.

\section{Disclosure Statement}

None declared.

\section{References}

1) Yamamoto H, Okada M, Takada $M$, et al. Videoassisted thoracic surgery through a single skin incision. Arch Surg 1998; 133: 145-7.

2) Rocco G, Martin-Ucar A, Passera E. Uniportal VATS wedge pulmonary resections. Ann Thorac Surg 2004; 77: 726-8.

3) Jutley RS, Khalil MW, Rocco G. Uniportal vs standard three-port VATS technique for spontaneous pneumothorax: comparison of post-operative pain and residual paraesthesia. Eur J Cardiothorac Surg 2005; 28: 43-6.

4) Son BS, Kim do H, Lee SK, et al. Small singleincision thoracoscopic surgery using an anchoring suture in patients with primary spontaneous pneumothorax: a safe and feasible procedure. Ann Thorac Surg 2015; 100: 1224-9.

5) Tsuboshima K, Wakahara T, Matoba Y, et al. Singleincision thoracoscopic surgery using a chest wall pulley for lung excision in patients with primary spontaneous pneumothorax. Surg Today 2015; 45: 595-9.

6) Song IH, Lee SY, Lee SJ. Can single-incision thoracoscopic surgery using a wound protector be used as a first-line approach for the surgical treatment of primary spontaneous pneumothorax? A comparison with three-port video-assisted thoracoscopic surgery. Gen Thorac Cardiovasc Surg 2015; 63: 284-9.

7) Ocakcioglu I, Alpay L, Demir M, et al. Is single port enough in minimally surgery for pneumothorax? Surg Endosc 2016; 30: 59-64. 